\title{
Comparative Study of Local SAD and Dynamic Programming for Stereo Processing Using Dedicated Hardware
}

\author{
John Kalomiros ${ }^{1}$ and John Lygouras ${ }^{2}$ \\ ${ }^{1}$ Department of Informatics and Communications, Technological Educational Institute of Serres, Terma Magnisias, 62124 Serres, Greece \\ ${ }^{2}$ Section of Electronics and Information Systems Technology, Department of Electrical Engineering \& Computer Engineering, \\ School of Engineering, Democritus University of Thrace, 67100 Xanthi, Greece
}

Correspondence should be addressed to John Kalomiros, ikalom@teiser.gr

Received 13 July 2009; Revised 2 October 2009; Accepted 30 November 2009

Recommended by Liang-Gee Chen

The processing results of two stereo accelerators implemented in reconfigurable hardware are presented. The first system implements a local method to find correspondences, the sum of absolute differences, while the second uses a global approach based on dynamic programming. The basic design principles of the two systems are presented and the systems are tested using a multitude of reference test benches. The resulting disparity maps are compared in terms of rms error and percentage of bad matches, using both textured and textureless image areas. A stereo head is developed and used with the accelerators, testing their ability in a real-world experiment of map reconstruction in real-time. It is shown that the DP-based accelerator produces the best results in almost all cases and has advantages over traditional hardware implementations based on local SAD correlation.

Copyright (C) 2009 J. Kalomiros and J. Lygouras. This is an open access article distributed under the Creative Commons Attribution License, which permits unrestricted use, distribution, and reproduction in any medium, provided the original work is properly cited.

\section{Introduction}

Real-time stereo vision is used in robot navigation, object recognition, environmental mapping, virtual reality, and so forth. The purpose of stereo processing algorithms is to find corresponding points in images acquired by a system of two or multiple cameras. Once reliable correspondences between image pixels are established, the problem of depth extraction is solved by triangulation [1].

Stereo algorithms can be classified into either local or global methods of correspondence [2]. Local methods match one small region around a pixel of interest in one image with a similar region in the other image by searching along epipolar lines. Typical similarity metrics used in local methods are the normalized cross-correlation (NCC), and the sum of squared differences (SSD) with its variation the sum of absolute differences (SAD), which is often used for computational efficiency. SSD and SAD find correspondences by minimizing the sum of squared or absolute intensity differences in small windows along epipolar lines. Local methods can be efficient but they are sensitive to noise and to local ambiguities, like occlusion regions or regions of uniform intensity.
Global methods compute disparities over a whole scan line or a whole image by minimizing a global cost function. They provide a best solution for the correspondence problem and minimize wrong matches at regions difficult to be matched locally [3]. They compute dense disparity maps of good quality but are computationally expensive and are seldom applied in real-time implementations. Commonly used global algorithms for stereo matching are based on dynamic programming (DP) $[4,5]$. The method consists of two phases, the cost matrix building phase and the backtracking phase, where the actual disparities are computed.

Real-time dense stereo is difficult to be achieved with general purpose serial processors and is often implemented using dedicated hardware, like Digital Signal Processors (DSPs), Graphics Processing Units (GPUs), and Application Specific Integrated Circuits (ASICs). Several systems are prototyped targeting Field Programmable Gate Arrays (FPGAs). Gate arrays are reconfigurable devices and represent an efficient solution for accelerating complex image processing functions, because they are based on a structure of small logic circuits that allows parts of an algorithm to be processed in parallel. 
This paper presents the basic design and processing results obtained by two different hardware accelerators dedicated to stereo matching. Both systems are implemented using Field Programmable Gate Arrays (FPGAs). The first is a stereo processor based on correlations between local windows, using the typical SAD metric. The other is based on global matching, applying a hardware-efficient variation of a dynamic programming (DP) algorithm. The choice to parallelize and comparatively evaluate SAD and DP in hardware is straightforward. SAD employs a hardwarefriendly metric of similarity and exploits the intrinsic parallelism of comparisons between local windows $[6,7]$. The main processing pipeline can be also used in order to implement other local methods of correspondence based on data-parallel window correlation. On the other hand, DP is a method commonly used for semiglobal optimization along a whole scanline. Its recursive cost plane computations are challenging to map in hardware because they lack inherent parallelism. When implemented in software the DP algorithm produces better disparity maps than SAD but it is much slower and difficult to perform in real-time. Although DP is more demanding than SAD to parallelize, it can be more straightforward and less expensive than other global methods like belief propagation or graph cuts.

A novel technique is presented in this paper for the parallelization of DP cost matrix computations within a predetermined disparity range. In both SAD and DP systems, matching is performed along epipolar lines of the rectified stereo pair. In both systems, matching cost is aggregated within a fixed $3 \times 3$ window using the intensity difference metric. In addition to plain SAD a hardware implementation of left-right consistency check is presented and a hardware median filter is used to enhance the disparity map. The system implementing dynamic programming is also enhanced by incorporating interscanline support. Both systems can process images in full VGA resolution and are able to produce 8-bit dense disparity maps with a range of disparities up to 64 levels. Both hardware designs are appropriate for real-time stereo processing, nominally producing hundreds of frames per second. However, they differ considerably in their basic design principles and in the quality of the final disparity maps.

For the assessment of the systems a hardware/software platform is developed, which is suitable to prototype and test image processing functions. The assessment of the two systems is performed using a number of reference images from the "Middlebury set", by comparing to the ground truth. A carefully adjusted stereo head developed in the laboratory is also used for real-time scene reconstruction, by extracting appropriate image features from the stereo pair.

The paper is organized as follows. In Section 2 a description of SAD and the proposed version of dynamic programming stereo algorithm is given. The hardware design of both systems is presented. In Section 3 the hardware/software platform used to prototype and assess the accelerators is presented. In Section 4 many reference images are processed and the results produced by the hardware accelerators are compared. The quality of the disparity maps is evaluated in terms of bad matches and rms error by comparing to the ground truth. Regions with rich texture as well as textureless regions of the images are used for this purpose. In Section 5 the two systems are compared in a real-world mapping experiment. Section 6 is a comparison with other systems found in the literature and Section 7 concludes the paper.

\section{Hardware Principles of SAD and DP Stereo}

2.1. SAD Algorithm and Hardware Principles. SAD represents a wide range of techniques that find correspondences by comparing local windows along epipolar lines in left and right images of the stereo pair [2]. It has been implemented in recent $[6,7]$ and early hardware systems for real-time stereo $[8,9]$ and has the advantage of a particularly simple and hardware-friendly metric of similarity, namely, the sum of absolute differences:

$$
\sum_{u, v}\left|I_{1}(u+x, v+y)-I_{2}(u+x+d, v+y)\right| .
$$

$I_{1}$ and $I_{2}$ refer to intensities in the left and right image, $(x, y)$ is the central pixel in the first image, $(x+d, y)$ is a point on the corresponding scanline in the other image displaced by $d$ with respect to its conjugate pair, and $u, v$ are indices inside the window. The point that minimizes the above measure is selected as the best match. This metric reduces hardware requirements only to additions and subtractions between intensities in the local windows.

While this method requires laborious search along epipolar lines in serial software implementations, it can be parallelized easily in hardware, allocating parallel comparisons between local windows to a number of processing elements. The block diagram of our system is shown in Figure 1. Window-level parallelism is applied across 32 and up to 64 levels of disparity, as shown in Figure 1, for a $3 \times 3$ window. The main processing element is shown in Figure 2 for a simplified case where comparisons are between 3-pixels in line1 of image1 and 3-pixels on the same line in image2. The shift between pixels and lines in order to form appropriate windows is achieved using delay lines. For example, a shift of a whole scanline between pixels in a rectangular window needs a 640-pixel-deep shift register, for an image with resolution $640 \times 480$. A number of $D$ processing elements are needed for $D$-levels of disparity.

After the $D$ SAD values are produced in parallel, their minimum value is found, using an array of parallel comparators that produce the minimum SAD in one clock cycle. A fully parallel implementation of this stage needs $D \times D$ comparators and demands a lot of hardware resources. However, it can also be implemented in several stages grouping together a number of SAD values and pipelining their output to the next stage of minimum derivation. This hardware derivation of minimum values is central in our implementation of SAD and is also used efficiently in our implementation of DP.

A tag index numbered from 0 to $D$ is attributed at each pixel in the search region. Among all $D$ pixels in the search region one pixel wins, corresponding to the minimum SAD value. The tag index of the winning pixel is 


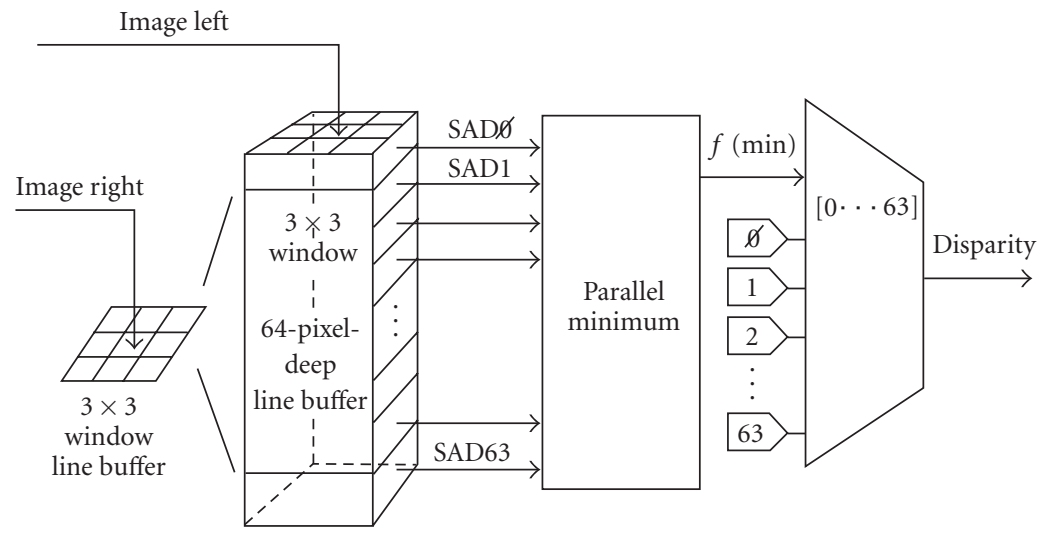

FIgURE 1: Parallel comparisons between $3 \times 3$ windows in left and right image, in a hardware implementation of the SAD algorithm.

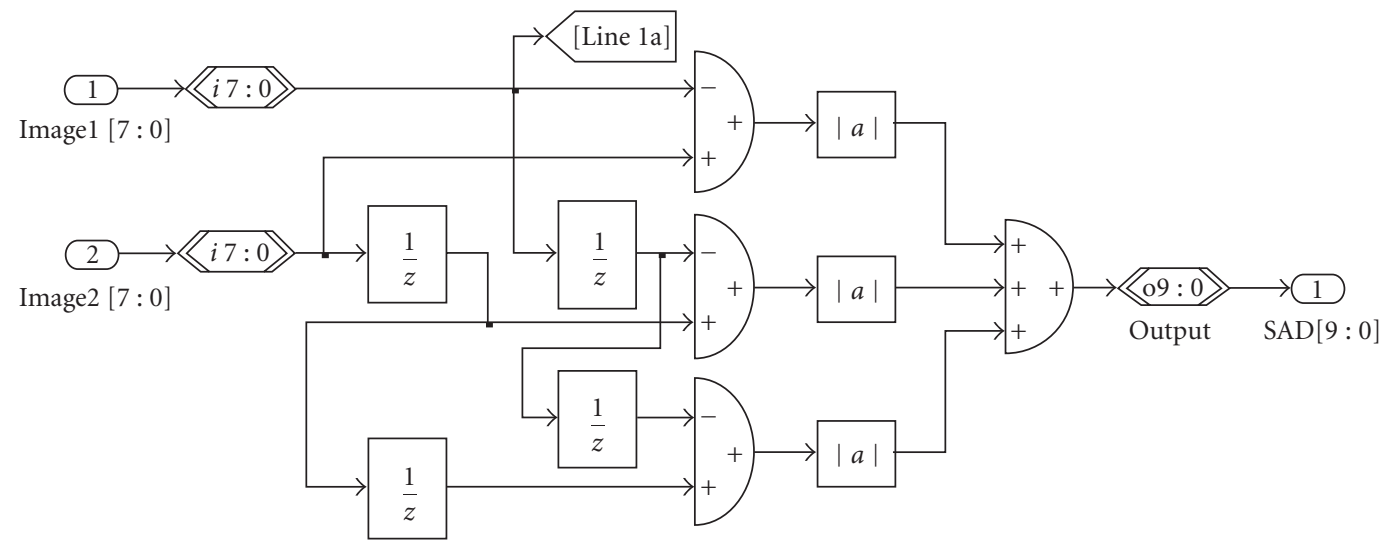

Figure 2: A simplified version of the basic processing element in the parallel computation of SADs.

derived and equals the actual disparity value. Details of this implementation can be found in [10].

2.2. Left-Right Consistency Check. An enhancement of the above system was designed in order to implement leftright consistency check. Pixels passing the consistency check are high confidence matches while those failing the test are considered bad matches or belong to occluded regions. SAD-based left-right consistency check can be implemented in hardware using an additional block for minimum SAD computations, as shown in Figure 3. Blocks in the first row output disparities with reference to the right image of the stereo pair. Each right pixel $B$ is compared with all left pixels $A^{\prime}-C^{\prime}$ hosted in the delay line, as shown in Figure 4(a). Blocks in the lower stages of Figure 3 output disparities with respect to the left image. In order to compare a left pixel with all candidate right pixels in the range of disparities it is imperative to mirror scanlines and reverse their ordering, as shown in Figure 4(b). In this way all candidate right pixels $C-A$ are hosted in the delay line when left pixel $B^{\prime}$ arrives for comparison.

The mirroring effect can be produced using on-chip RAM memory. Each $N$-pixel-wide scanline is written into memory in the first $N$ clock cycles and is read out of memory in a Last-In First-Out (LIFO) manner. LIFO-1 memory blocks in Figure 3 are implemented as pairs of dual-port RAM blocks. An even scanline is read from RAM-2 while an odd scanline is written in RAM- 1 at the same time. RAM blocks alternate their read/write state every next scanline. In this way, streaming scanlines are mirrored at the output of LIFO-1 blocks at clock rate.

In order to compensate for the mirroring function applied in the input stage of the lower left- right comparison blocks, a LIFO-2 memory block is used at the output of the right-left comparison blocks. In this way both disparity maps are in phase.

Median filtering of the output disparity maps can substantially improve the final result. Figure 5 shows part of our hardware mapping of a $3 \times 3$ median filter designed for this purpose. Pixels are ordered by pairwise comparisons in nine subsequent steps and the median is selected.

The final consistency check between left-and rightreferenced disparity images is performed by a comparator unit, as shown in Figure 6. The 32-pixel active range of left-referenced disparities is hosted in a taps unit, while a multiplexer selects the disparity element corresponding to the current right-referenced disparity value. Left and right disparities are compared and if found equal, the disparity 


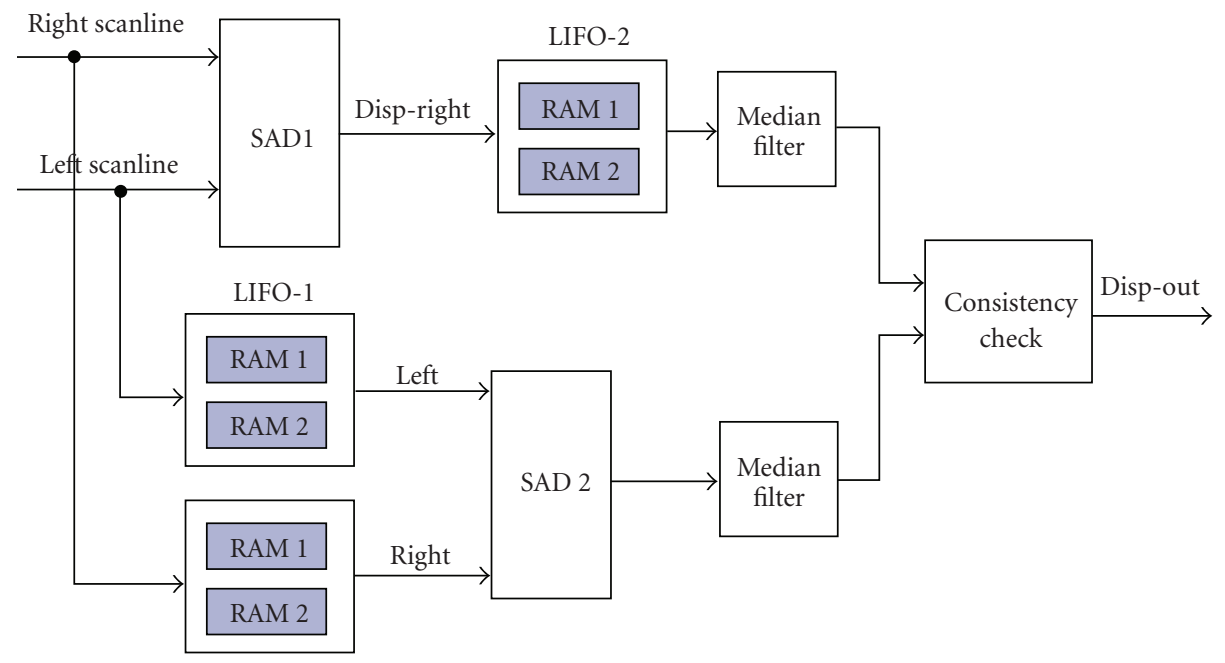

FIGURE 3: Block diagram of the SAD-based system with left-right consistency check.

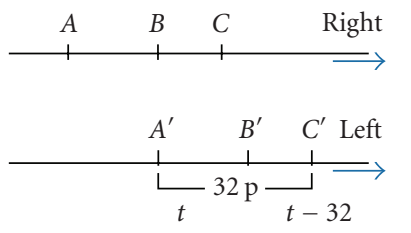

(a)

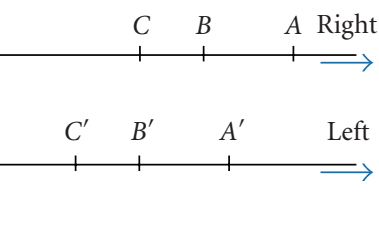

(b)
Figure 4: (a) Pixel $B$ on the right scanline is compared for similarity with 32-pixels $A^{\prime}-C^{\prime}$ of the left scanline stored in the delay line. (b) Scanlines are mirrored for consistency check: Pixel $B^{\prime}$ on the left scanline is compared with all 32-pixels $\mathrm{C}-\mathrm{A}$ on the right scanline.
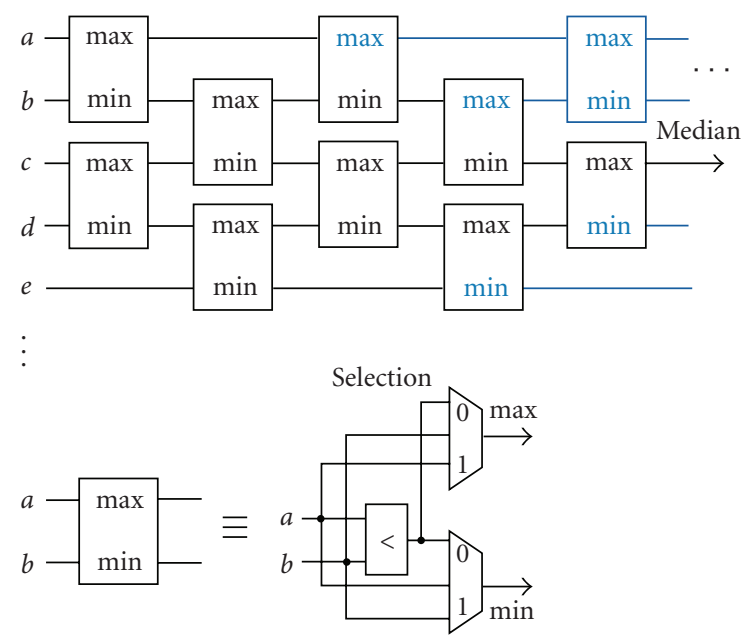

FIGURE 5: Ordering circuit for the implementation of a median filter. Elements shown in blue are redundant for the selection of the median.

value is put out. If the two values differ, we transmit the last left-referenced disparity value in order to correct occlusion boundaries or bad matches. Significant corrections at occlusion boundaries are found using this method.
Processing results using the SAD hardware accelerator are presented in Section 4.

2.3. DP Algorithm and Hardware Principles. Dynamic programming for stereo is mathematically and computationally more complex than local correlation methods, since stereo correspondence is derived as a globally optimum solution for the whole scanline [4]. Our hardware system is designed as a fully parallel implementation of a variation of the Cox method for maximizing likelihood [5]. The algorithm is developed in two phases, namely, the cost plane building phase and the backtracking phase. The cost plane is computed as a two-dimensional matrix of minimum costs, one cost value for each possible correspondence $I_{i} \leftrightarrow I_{j}$ between left and right image intensity values, along a scan line. One always proceeds from left to right, as a result of the ordering constraint [11]. This procedure is shown in Figure 7 , where each point of the $2 D$ cost function is derived as the minimum transition cost from the three neighbouring cost values. Transition costs result from previous costs, adding a matching or occlusion cost, $s_{i j}$ or occl, according to the following recursive procedure:

$$
\begin{aligned}
C(i, 0)=i \times \text { occl, } \\
C(0, i)=i \times \text { occl, } \\
C(i, j)=\min \left\{C(i-1, j-1)+s_{i j}, C(i-1, j)\right. \\
\quad+\text { occl, } C(i, j-1)+\text { occl }\} .
\end{aligned}
$$

In the above equations, the matching cost $s_{i j}$ is minimized when there is a match between left and right intensities. The following dissimilarity measure was used based on intensity differences:

$$
s_{i j}=\frac{\left(I_{l}(i)-I_{r}(j)\right)^{2}}{\sigma^{2}},
$$




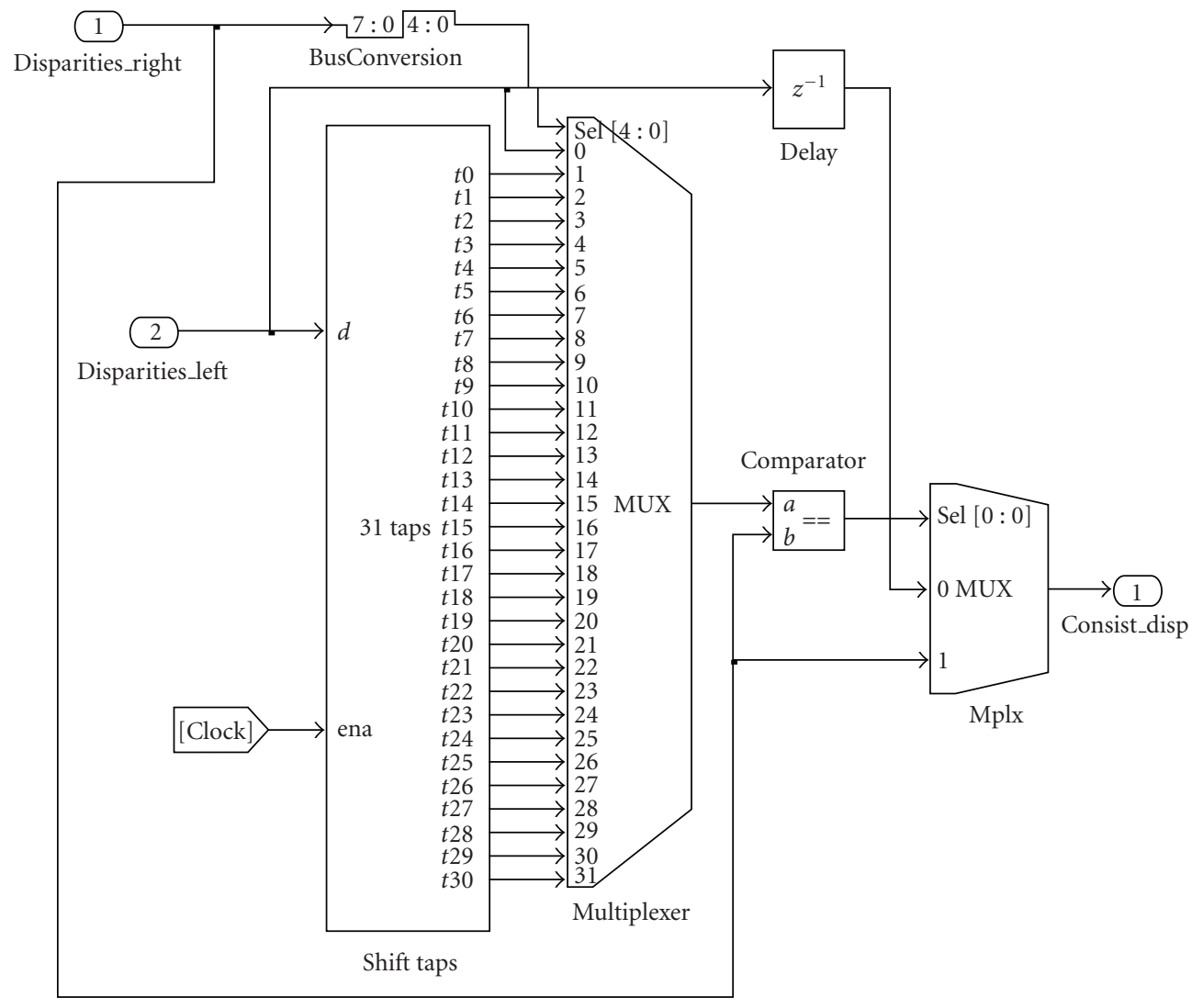

Figure 6: Implementation of the left-right consistency check.

where $\sigma$ represents the standard deviation of pixel noise. Typical values are $\sigma=0.05-0.12$ for image intensities in the range $[0,1]$. In our implementation we calculate $s_{i j}$ within a fixed $3 \times 3$ window applied in both images. The occlusion cost occl is the cost of pixel $j$ in the right scanline being skipped in the search for a matching pixel for $i$ and in our tests takes a value occl $=0.2$.

According to (2)-(4), the cost matrix computation is a recursive procedure in the sense that for each new $\operatorname{cost} C(i, j)$ the preceding costs on the same row and column are needed. In turn, previous costs need their precedent costs, rendering the parallel computation of costs an intractable task. In order to parallelize the cost matrix computation in hardware, we design a novel variation of the cost matrix computations, using an adequate slice of the cost matrix along the diagonal of the cost plane, as shown in Figure 7. Working along the diagonal allows a subset of $D$ cost states to result in parallel from the preceding subset of cost states, in step with the input stream of left and right image scanlines. Figure 7 shows a slice along the diagonal supporting a maximum disparity range of 9-pixels. Starting from a known initial state (here $\mathrm{C}_{1 A}, \mathrm{C}_{00}$, $C_{2 A}, C_{2 B}, C_{2 C}, C_{2 D}, C_{2 E}, C_{2 F}, C_{2 G}$ ) lying on the axes and given by (2) and (3)), it is possible to calculate all states in the slice, up to the end of the cost plane, following the diagonal. This computation is performed at each computation cycle by setting as a next input the output computed in the previous step. Figure 8 shows the cost matrix computation more analytically. By taking three input states together and adding occlusion or matching costs, the processing element computes the cost of the diagonal, vertical and horizontal path to each adjacent point. These costs are taken together and the minimum value is produced by an appropriate parallel-computation stage. Tag values are attributed to all three possible paths. A tag " 1 " is attributed to the vertical path, a tag "0" is attributed to diagonal paths, while a tag value " -1 " is attributed to the horizontal path.

Wining tags at each point are stored in RAM memory and are read in reverse order during backtracking, in order to follow backwards the optimum path. RAM is written during the cost-computation phase and is read during the backtracking stage. The same LIFO RAM blocks used in Figure 3 for mirroring the scanlines are also used here in order to implement the backtracking stage. A number of $D$ RAM blocks are needed, where $D$ represents the useful disparity range (nine in the case of the state machine in Figure 8). Each RAM block has a depth of $N$ cells, where $N$ represents the length of scanline. All RAM cells are only 2-bit wide, since they store the values $-1,0,1$.

Stored tag values are used to calculate the change in the disparity value per pixel during the backtracking phase. The following rules are applied for the disparity computations during backtracking.

Starting at the end of the cost plane $(N, N)$, corresponding stored tags are examined. The case of tag $=$ " 1 " 


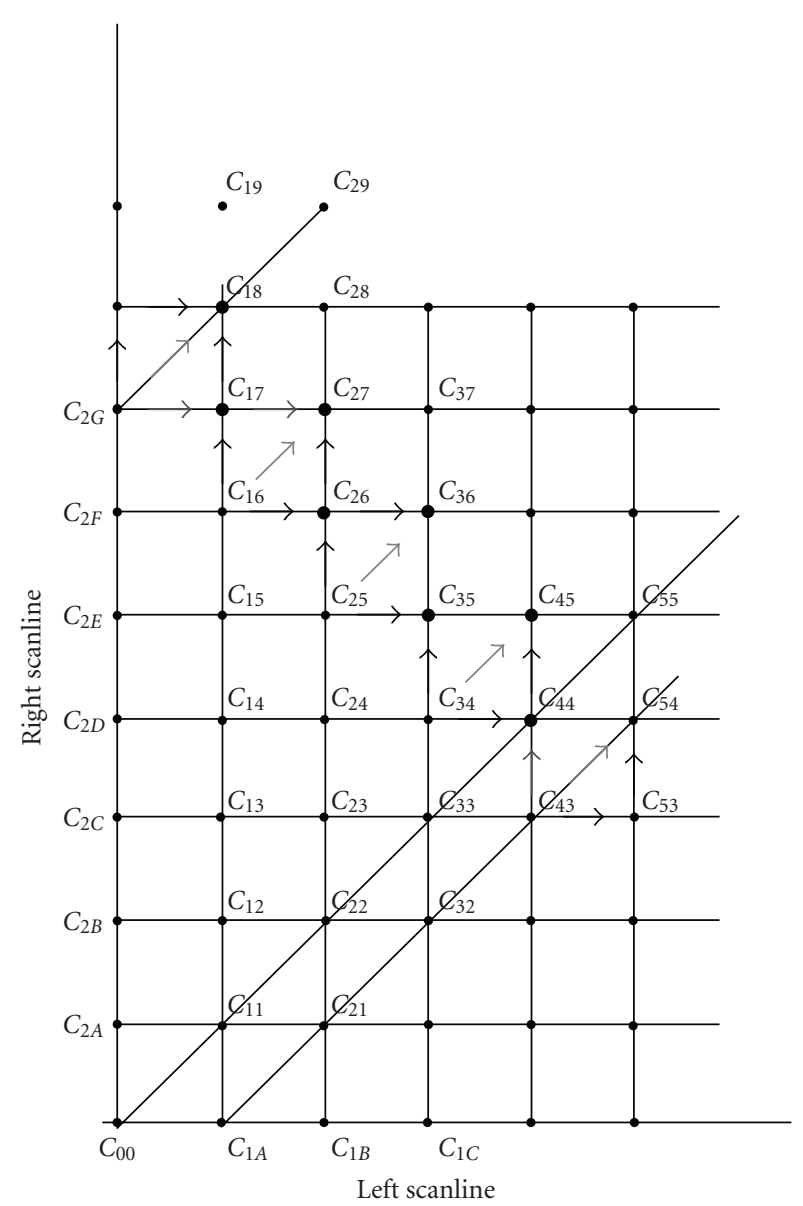

FIGURE 7: Cost plane and a slice along the diagonal. Nine states are computed in parallel in this example.

corresponds to skipping a pixel in the left scanline and to a unit increase in disparity. The case of tag $=$ " -1 " corresponds to skipping a pixel in the right scanline and means a unit decrease in disparity, while the case of tag $=$ " 0 " matches pixels $(i, j)$ and therefore leaves disparity unchanged. Beginning with zero disparity, the minimum cost path is followed backwards from $(N, N)$, and the disparity is tallied, until point $(1,1)$ is reached.

The above rules have been mapped in hardware by arranging tag values in vertical columns corresponding to the columns of the cost matrix in Figure 7. The main idea is shown in Figure 9, where each column of $D$ tag values corresponds to one pixel of disparity. All elements in a column are indexed starting from the bottom on the diagonal. Entry and exit indices in the columns of Figure 9 trace the path of minimum cost. In the proposed implementation $D$ parallel stages are used and DP backtracking rules are applied to find the "exit" index for each column in one clock cycle. This index is derived as the minimum of all indices in the column that correspond to a tag value that is either " 0 " or " -1 ". Upon exiting the previous column we enter the next by moving either diagonally one step to the bottom (in the case of tag $=0$ ) or horizontally ( $\operatorname{tag}=-1$ ). If we consider exit to be the index of the exit point from the $(i-1)_{\text {th }}$ column and entry to be the index of the entry point to the $i_{\text {th }}$ column, then the change in disparity at pixel $i_{\text {th }}$ is found using the equation:

$$
\triangle d=\text { exit-entry. }
$$

Starting with $d=0$, the system tracks disparities adding $\Delta d$ at each step.

A block diagram of the overall system is shown in Figure 10.

2.4. Interscanline Support in the DP Framework. The system described above uses a two-dimensional cost plane and optimizes scanlines independently. The problem can expand as a search for optimal path in a 3D search space, formed as a stack of cost planes from adjacent scanlines. The cost of a set of paths is now defined as the sum of the costs of individual paths.

A system with support from adjacent scanlines is implemented and cost states are computed using in the place of (4) the following relation:

$$
\begin{aligned}
C(i, j)=\min \left\{\frac{1}{k_{\max }} \sum_{1}^{k_{\max }}\left[C_{k}(i-1, j)+\mathrm{occl}\right],\right. \\
\frac{1}{k_{\max }} \sum_{k=1}^{k_{\max }}\left[C_{k}(i, j-1)+\mathrm{occl}\right], \\
\left.\frac{1}{k_{\max }} \sum_{k=1}^{k_{\max }}\left[C_{k}(i-1, j-1)+s_{k i j}\right]\right\},
\end{aligned}
$$

where $k$ is the scanline index and $k_{\max }$ is the maximum number of adjacent scanlines contributing in the costcomputation.

Just like in the case of using windows for cost aggregation, the underlying hypothesis here is that adjacent points on the same image column are connected and belong to the same surface or object boundary. This additional interscanline constraint provides more global support and can result in better matching.

In order to provide support from one adjacent scanline, the proposed system buffers the preceding scanline in a delay line and streams both current and former scanlines through the cost-building state machine. The cost-computation stage is now enhanced according to (7) in order to produce cost states for both streaming scanlines. Minimum costcomputation and memory stages are implemented by the same circuits as in the plain system. More scanlines can be added by expanding the design in the same way.

Processing results using the DP hardware accelerator are shown in Section 4.

\section{Hardware/Software Codesign For System Assessment}

In order to assess the performance of the stereo accelerators described in Section 2 a host/coprocessor architecture is 

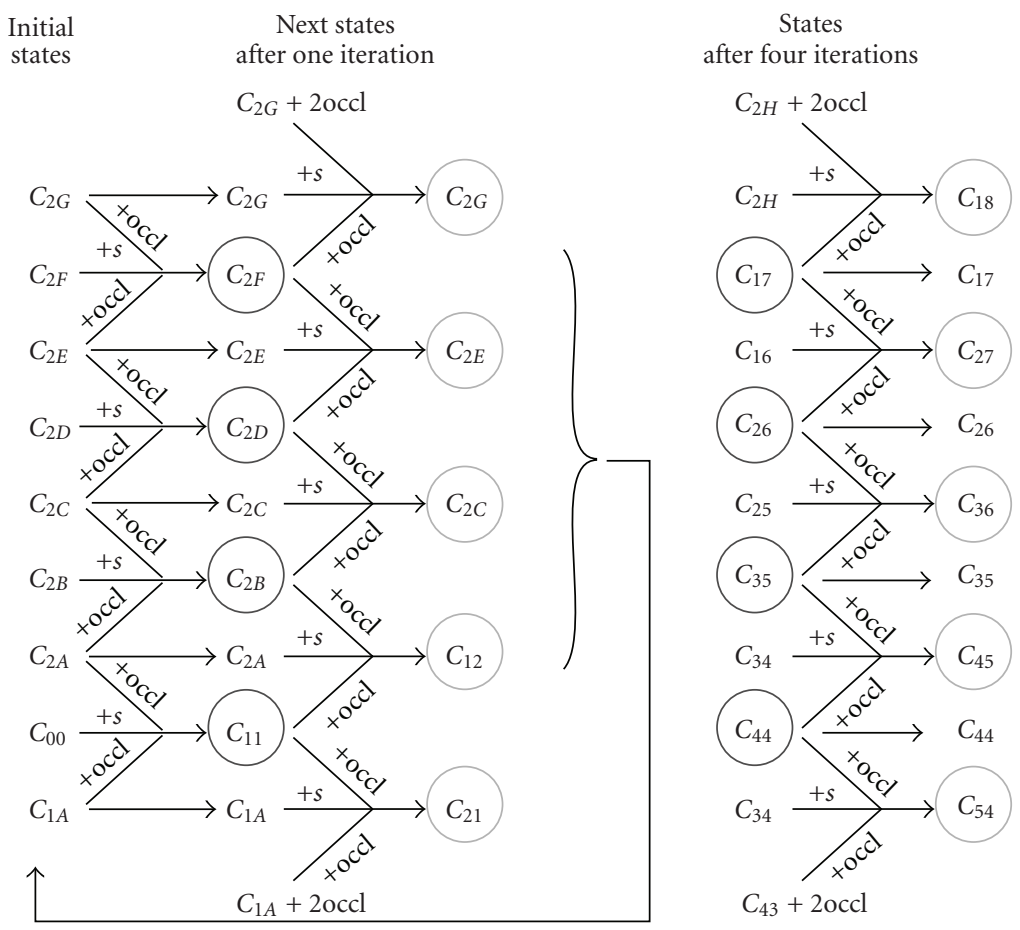

FIGURE 8: Initial state and successive derivation of next states with a nine-state processing element.

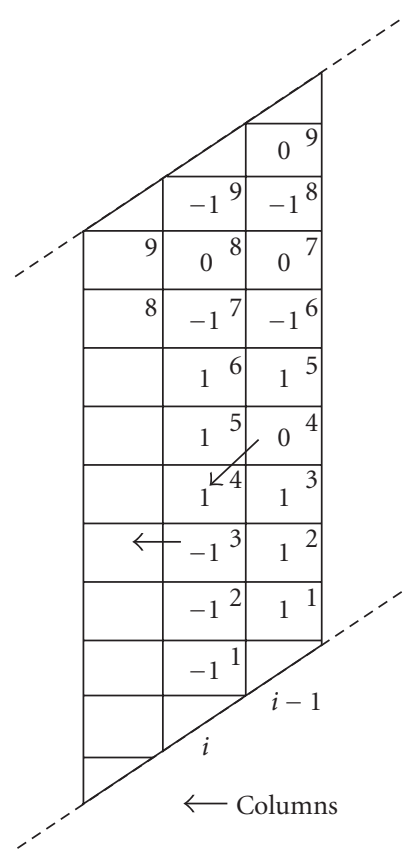

Figure 9: Tag values $(-1,0,1)$ arranged in columns and indexed from 1 to $D$ (here $D=9$ ) for the backtracking stage. Arrows indicate exit and entry in successive columns.

developed. On the coprocessor side a Cyclone II FPGA prototyping board, made by Altera Corporation, is used. This board features a Cyclone II 2C35 medium-scale FPGA device with a total capacity of 33000 logic elements and 480000 bits of on-chip memory. Apart from the stereo accelerator, we use a Nios II embedded processor for data streaming and control operations and a number of peripheral controllers in order to interface with external memory and communication channels. A block diagram of the embedded system is shown in Figure 11.

On the host part a vision system is implemented, appropriate for a spectrum of industrial applications. The host computer features an on-board high speed USB 2.0 controller and a NI 1408 PCI frame grabber. The host application is a LabVIEW virtual instrument (VI) that controls the frame grabber and performs preprocessing of the captured images. The frame grabber can support up to five industrial cameras performing different tasks. In our system, the VI application captures a pair of frames with resolution up to $640 \times 480$-pixels from two parallel CCIR analog B\&W CCD cameras (Samsung BW-2302). Figure 12 is a picture of the stereo head along with the accelerator board.

The LabVIEW host application communicates with the USB interface and transmits a numeric array out of the captured frames. An advantage of using LabVIEW as a basis for developing the host application is that it includes a VISION library able to perform fast manipulation of image data.

When the reception of the image array is completed at the hardware board end, the system loads the image data to the dedicated stereo accelerator and sends the output to a VGA monitor. Alternatively, the output is sent back to the host application via the USB 2.0 channel for further processing. The procedure is repeated with the next pair of captured frames. 


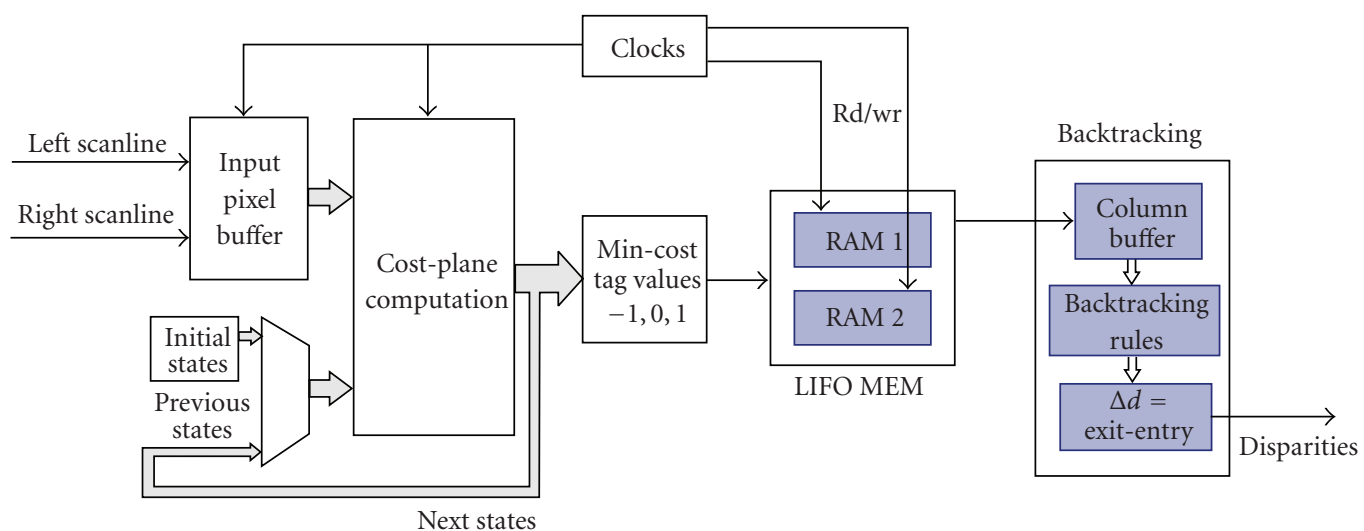

FigURE 10: Block diagram of the stereo system based on dynamic programming.

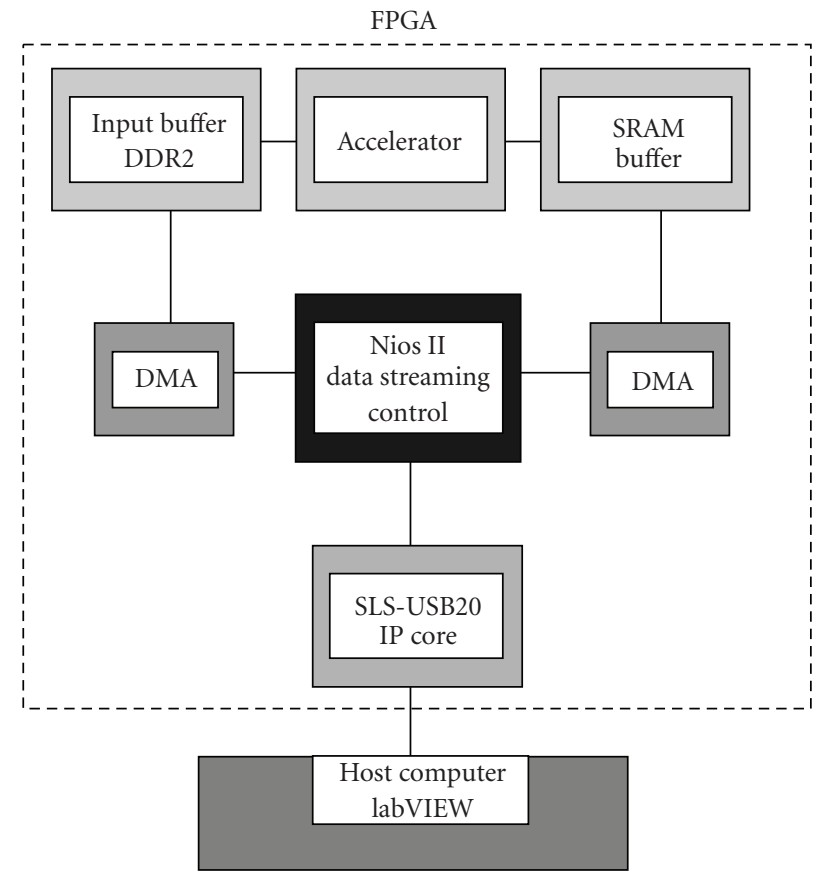

FIGURE 11: Hardware platform for the evaluation of the processors: system-on-a-chip and communication with host.

Typical images captured in the laboratory and the resulting depth maps produced by hardware are presented in Section 4.

\section{Evaluation of SAD and DP Systems}

Both SAD and DP accelerators are able to process up to 64 levels of disparity and produce $640 \times 4808$-bit depth maps at clock rate. Using appropriate pipelining between stages the SAD processor can be clocked at $100 \mathrm{MHz}$ which is the maximum frequency allowed by the on-board oscillator. The system outputs disparity values at a rate of $50 \mathrm{Mpixels} / \mathrm{s}$ and processes a full VGA frame at a nominal time of $6.1 \mathrm{~ms}$. This is equivalent to 162 frames/s. The DP-based system has more strict timing requirements because it uses feedback loops, as for example in the cost matrix computation stage. The higher possible frequency for our present implementation is $50 \mathrm{MHz}$. A pair of $640 \times 480$ images is processed at $12.28 \mathrm{~ms}$, which is equivalent to 81 frames/s or $25 \mathrm{Mpixels} / \mathrm{s}$. However, in a "normalized" sense both accelerators have the same disparity throughput, since hardware optimization can potentially resolve the timing issue. The reported throughput of $50 \mathrm{Mpps}$ is high and suitable for demanding real-time applications, like navigation or environmental mapping. Table 1 presents timing requirements and nominal frame rates for both systems. In practice, apart from the accelerator throughput, the frame rate depends also on the camera type and other system parts. 


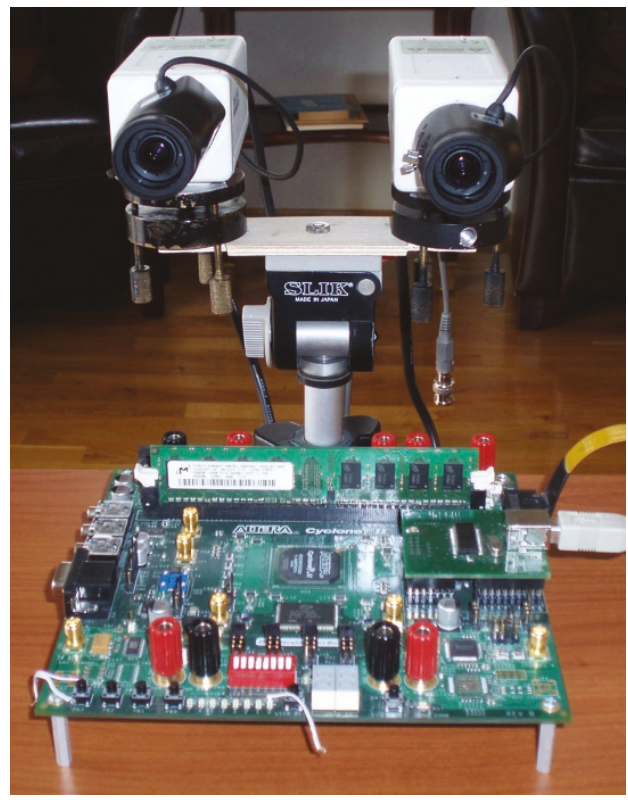

FIGURE 12: The stereo head with the FPGA board.

Table 2 shows the average resources required from a Cyclone II EP2C35 FPGA device in order to implement the designs presented in Section 2 as FPGA processors. The number of logic elements (LE) is given, along with necessary on-chip memory bits. The table refers to the plain processors and their enhancements described in Sections 2.2 and 2.4, that is, SAD-based left-right consistency check and interscanline support for DP processing. In addition to the resources needed for plain SAD implementation, left-right consistency check requires RAM memory for the implementation of the LIFO mirroring blocks. The DP accelerator requires onchip memory for the storage of minimum cost tag values, but can be implemented with fewer logic elements than $\mathrm{SAD}$, allowing for larger disparity range. Nios II processor and peripheral controllers require an additional overhead of about 7000 LEs and 160000 bits of embedded memory. In the last column of Table 2 the equivalent gate count is given and can be used for comparison with ASIC stereo implementations. Gate count is inferred from the fitting result of the design compiler and represents maximum resource utilization within each logic element.

Increasing the range of disparities increases proportionally the necessary resources for both SAD and DP architectures. Applying block reusing techniques could optimize resource usage, but on the expense of processing speed. Increasing image resolution has little effect on the resources needed for SAD, since only two image lines are stored in onchip memory, in order to form the $3 \times 3$ window. However, in our present DP system, increasing the length of scanline increases proportionally the memory needed for the storage of tag values.

Depth maps produced by the proposed SAD and DP hardware systems are evaluated using a variety of reference stereo datasets, produced in the laboratory by Scharstein and Szeliski [12, 13]. Pixel-accurate correspondence information is acquired using structured light and is available for all datasets. Also, the reference image series available from the Tsukuba University [14] is used in this evaluation.

First, processing results are presented using the plain version of the SAD and DP accelerators, without their enhancements. As it is explained in Section 2, in both cases the same intensity difference metric is used and matching cost is aggregated within a $3 \times 3$ window. Both systems are mapped in hardware using comparable resources. They both represent a fully parallel version of the underlying algorithm and produce disparities at clock rate. Also, they are assessed using the same FPGA platform. Comparative results are shown in Figure 13. Each row of Figure 13 presents the left image of the reference pair, the ground truth and the depth maps produced by the plain SAD and DP hardware systems, without their enhancements.

The main weaknesses of the two types of algorithms become evident in their FPGA implementation. As shown in Figure 13, in most cases SAD depth maps contain noise spots, especially in image areas of low light intensity. Processing results from the DP hardware show horizontal streaks caused by the horizontal transmission of errors along epipolar lines. This is because of the weak correlation between scanlines in the DP system, since this processor does not yet include interscanline support. In general, DP global matching is more accurate and produces fine detail as compared to SAD. However, in some images there is better object segmentation in the depth map produced by the SAD accelerator, as in the case of "bowling" and "sawtooth" images (Figure 13).

The quality measures proposed by Scharstein and Szeliski [15] which are based on known ground truth data $d_{T}(x, y)$ are used for further evaluation. The first measure is the RMS (root-mean-squared) error between the disparity map $d_{A}(x, y)$ produced by the hardware accelerator and the ground truth map:

$$
R=\left(\frac{1}{N} \sum_{x, y}\left|d_{A}(x, y)-d_{T}(x, y)\right|^{2}\right)^{1 / 2},
$$

where $N$ is the total number of pixels in the area used for the evaluation. RMS error is measured in disparity units. The second measure is the percentage of bad matching pixels, which is computed with respect to some error tolerance $\delta_{d}$ :

$$
B=\frac{1}{N} \sum_{(x, y)}\left(\left|d_{A}(x, y)-d_{T}(x, y)\right|>\delta_{d}\right) .
$$

For the tests presented in this paper a disparity error tolerance $\delta_{d}=1$ is used.

The above measures are computed over the whole depth map, excluding image borders, where part of the image is totally occluded. They are also computed over selected image regions, namely, textureless regions and regions with welldefined texture. In this way conclusive statistics are collected for both hardware processors, based on a variety of existing test benches.

Figure 14 is a comparative plot that presents the above statistical measures calculated over the whole image, for the 
TABLE 1: Processing speeds for the SAD and DP accelerators.

\begin{tabular}{lcccc}
\hline Type of implementation & Image resolution & Maximum achieved frequency $(\mathrm{MHz})$ & Normalized throughput $(\mathrm{Mpps})$ & Frame rate(fps) \\
\hline SAD & $640 \times 480$ & 100 & 50 & 162 \\
DP & $640 \times 480$ & 50 & 50 & 81 \\
\hline
\end{tabular}

TABLE 2: Typical resources needed for implementing SAD and DP systems in FPGA.

\begin{tabular}{|c|c|c|c|c|c|c|}
\hline Type of implementation & Image resolution & Levels of disparity & Logic elements & Memory bits & $\begin{array}{l}\text { 9-bit embedded } \\
\text { multipliers }\end{array}$ & $\begin{array}{c}\text { Equivalent gate } \\
\text { count }\end{array}$ \\
\hline SAD & $320 \times 240$ & 32 & 12200 & 12176 & - & 290000 \\
\hline SAD & $640 \times 480$ & 64 & 24300 & 27200 & - & 594000 \\
\hline SAD + consistency check & $320 \times 240$ & 32 & 23900 & 37760 & - & 630000 \\
\hline Median filter & $320 \times 240$ & - & 676 & 5088 & - & 33500 \\
\hline DP & $320 \times 240$ & 33 & 9300 & 71792 & 33 & 530000 \\
\hline DP & $640 \times 480$ & 65 & 21500 & 270240 & 66 & 1600000 \\
\hline $\begin{array}{l}\text { DP with support from } \\
\text { two adjacent scanlines }\end{array}$ & $320 \times 240$ & 33 & 15573 & 75088 & 66 & 740000 \\
\hline $\begin{array}{l}\text { Nios II processor }+ \text { on } \\
\text { chip memory }\end{array}$ & - & - & 2000 & 120000 & - & 520000 \\
\hline Other controllers & - & 一 & 5000 & 40000 & - & 260000 \\
\hline
\end{tabular}

SAD and DP hardware accelerators. The quality measures for textured regions are presented in Figure 15. The statistics of textureless regions are presented in Figure 16. We define textureless regions as image areas where the average horizontal intensity gradient is below a given threshold.

The error statistics presented in Figures 14, 15, 16 confirms in quantitative terms that the global matching performed by the DP hardware accelerator produces more reliable disparity maps than the block matching method used by the SAD processor. This appears to be true for both types of measures $(R$ and $B$ ) and for different kinds of image regions.

Next, results were obtained using the enhanced accelerators. First, the system performing SAD-based left-right consistency check was tested, using the "Tsukuba" and "cones" reference images. As explained in Section 2.2, the system produces consistent disparities with respect to the right image and replaces occlusions with the last measured left disparity. Figures 17 (b) and $17(\mathrm{~d})$ on the right are the depth maps produced by the system shown in Figure 3, while Figures 17(a) and 17(c) show for comparison the depth map produced by the plain SAD processor, shown in Figure 1. Matching at occlusion boundaries is visibly improved and the overall depth map contains less noise due to median filtering and replacement of bad matches.

The result produced by the enhanced version of the DP accelerator is shown in Figures 18(b) and 18(d), while the output of the plain DP system is shown for comparison in Figures 18(a) and 18(c). As explained in Section 2.4, this system has a multiple cost-computation stage, where the cost planes of three adjacent scanlines are processed in parallel and minimum cost is produced according to (7).
Taking into account a correlation between scanlines reduces the horizontal streaking effect that is inherent in the linebased global optimization. Attenuation of horizontal streaks is mainly visible around object boundaries, as can be seen in the "cones" depth map. Incorporating support from more scanlines can further improve the result, however it expands the design and can only be achieved by migrating to more dense devices.

Next, the quality measure given by (9) is used for the evaluation of the depth maps of Figures 17 and 18. The measures are applied over the whole image and the statistics presented in Figure 19 are obtained. The result reflects the visible improvements in the depth maps.

Some discussion is needed concerning the robustness of the above measures. RMS error and percentage of bad matches obviously depend on the available ground truth maps and the image area where they are applied. They represent an indication rather than definitive evidence of how "good" a stereo system is. Real stereo sensors work in the absence of ground truth and by processing a familiar real scene they can provide subjective evidence of the quality of a stereo system. In addition to the evaluation presented above, the stereo head shown in Figure 12 was used to capture and process real scenes. A typical result is shown in Figure 20. A captured image is followed by depth maps produced by the plain and enhanced SAD and DP-based processors. These results provide an additional quality measure for the proposed systems. As shown in Figure 20, depth maps produced by the SAD processors are dominated by noise spots, although median filtering and left-right consistency check can improve the result. The DP-based system produces smooth depth maps, accurate in most parts of the image. 

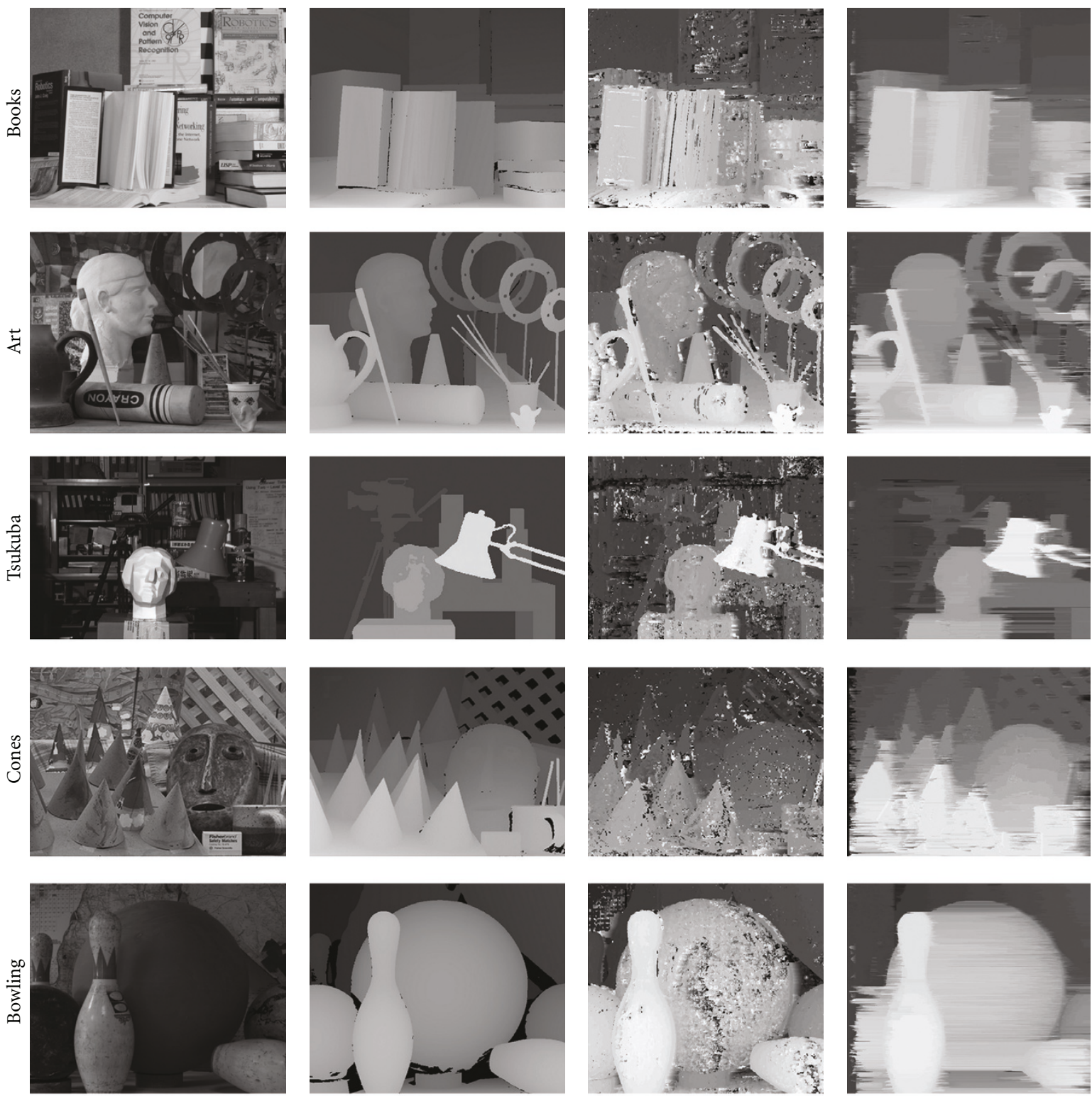

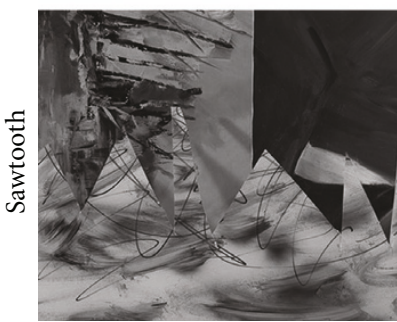

(a)

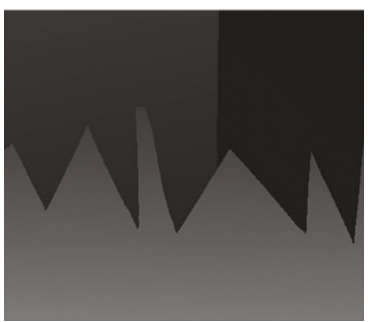

(b)

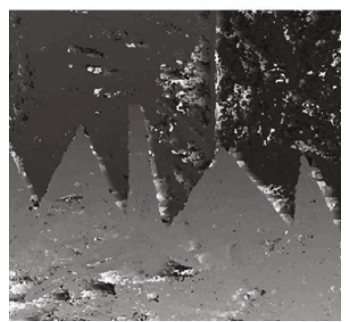

(c)

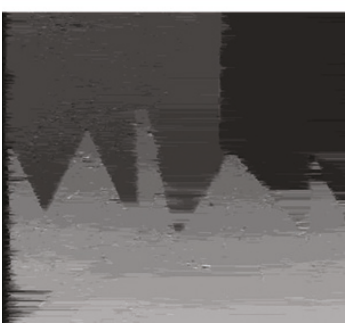

(d)

Figure 13: Processing of reference datasets. From top to bottom: Books, Art, Tsukuba, Cones, Bowling, and Sawtooth data-sets. (a) Reference test image, (b) ground truth, (c) processing by the SAD accelerator, and (d) processing by the DP accelerator.

Although some streaking effect is blurring object boundaries, the overall subjective result is satisfactory. Object boundaries can be refined by the same left-right consistency check described in the case of the SAD processor, with substantial cost in gate resources.
Migrating to larger FPGA devices can allow incorporating left-right consistency check as well as more interscanline support in the DP framework. However, the cost and performance of such a system should be compared with the cost of implementing other global techniques for stereo processing. 


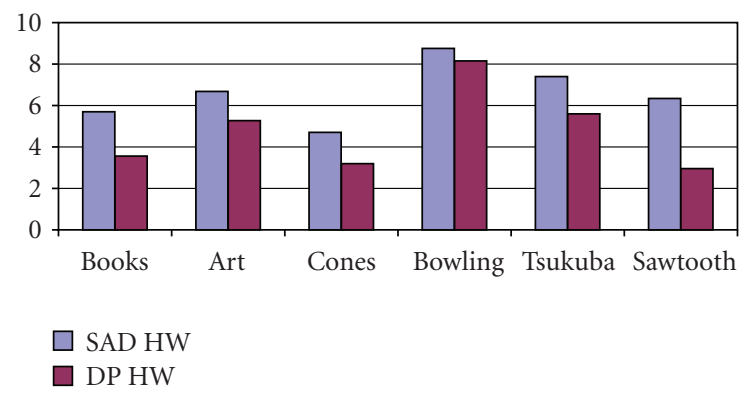

(a) Total image (rms error)

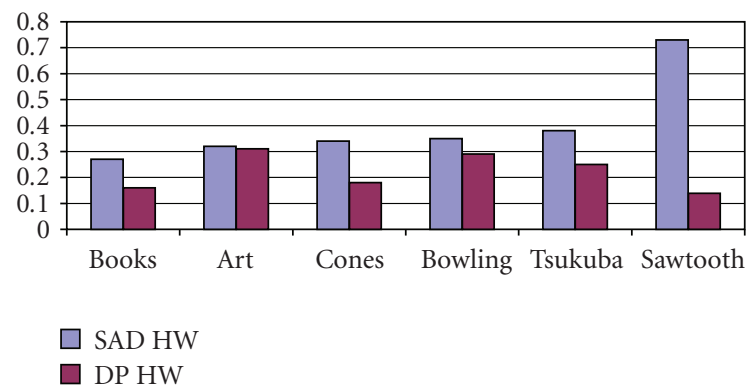

(b) Total image (bad matches)

FIGURE 14: Error statistics computed over the whole image. (a) rms error, (b) percentage of bad matching pixels.

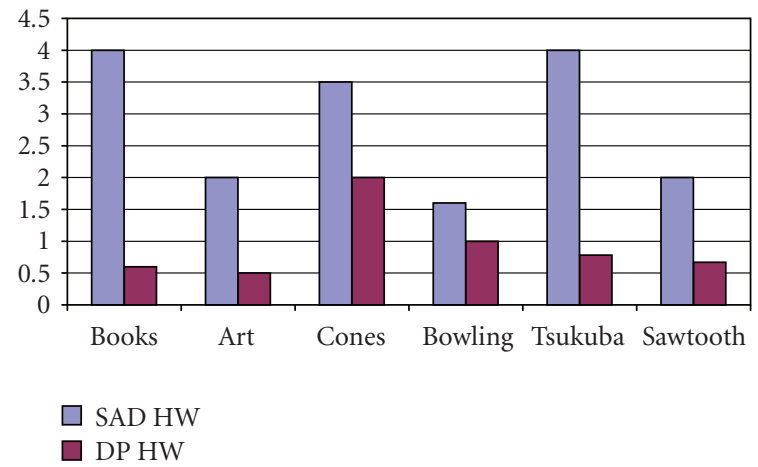

(a) Textured regions (rms error)

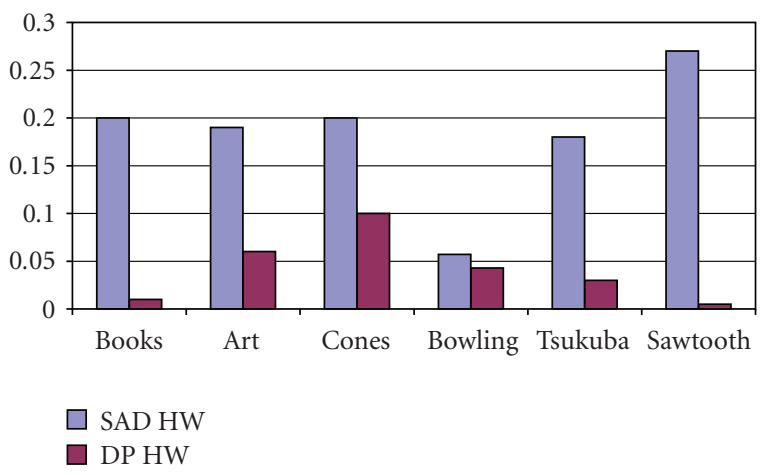

(b) Textured regions (bad matches)

FIGURE 15: Errors computed in textured regions for the various data-sets. (a) rms error, (b) percentage of bad matching pixels.

\section{Real-Time Scene Reconstruction with SAD and DP Systems}

In this paragraph the two systems are tested using a technique for real-time scene reconstruction, with the movable stereo head. A pair of gray-scale, 8-bit, left and right video sequences is captured, and the depth map is produced in real-time using the SAD and DP hardware, alternatively. A robust feature extraction algorithm is developed in software and is applied to process the captured frames in the time between data transfers to and from the FPGA board. The selected features are projected back to the world reference frame, using a suitable transformation from image pixel to real-world. At the end of the video sequence the real scene is reconstructed by a cloud of points resulting from the superposition of all projections from all video frames. The result is noisy and depends on the type of the selected features, however, it allows the hardware vision system to be applied and tested in a real-world experiment, suitable for robot navigation and mapping.

The feature extraction algorithm applied in this experiment can be summarized as follows

(a) Corners are extracted from both left and right video frames using the cross-section of horizontal and vertical edges. A thinning procedure is applied and the result is a pair of binary images with point features.

(b) For each feature position $\left(u_{L}, v_{L}\right)$ in the left image, we look for a matching feature in the right image using as a measure for correspondence the minimization of the Euclidean distance between features:

$$
E=\left(u_{L}-u_{R}-d\right)^{2}+\left(v_{L}-v_{R}\right)^{2},
$$

where $\left(u_{L}, v_{L}\right)$ is the feature position in the left image, $\left(u_{R}, v_{R}\right)$ is the feature position in the right image, and $d$ is the disparity $u_{L}-u_{R}$ of pixel $\left(u_{L}, v_{L}\right)$, as derived by the depth map.

The algorithm results in a limited number of matching features for each video frame. Depending on the error threshold $E$ allowed in (10) the result can be several tens of features per frame.

The feature position $\left(u_{L}, v_{L}\right)$ on the image is translated into a point $\left(X_{i}, Y_{i}, Z_{i}\right)$ in the frame of reference of the stereo head, as shown in Figure 21, applying the relations:

$$
\begin{aligned}
X_{i} & =\frac{f b}{\left(u_{L}-u_{R}\right)}, \\
Y_{i} & =\frac{1}{f} X_{i}\left(u_{L}-u_{0}\right)-\frac{b}{2}, \\
Z_{i} & =\frac{1}{f} X_{i}\left(v_{L}-v_{0}\right),
\end{aligned}
$$

where $f$ is the camera focal length measured in pixels, $b$ is the "baseline", that is, the distance between left and right camera focal axes, and $\left(u_{0}, v_{0}\right)$ is the center of the image measured in pixels. The focal length for both cameras is measured to be 


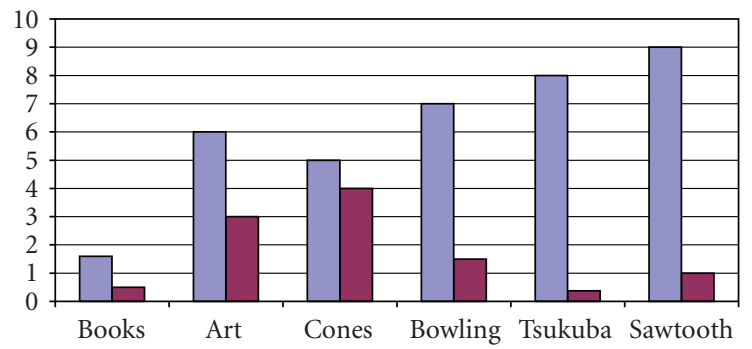

$\square$ SAD HW $\square$ DP HW

(a) Textureless regions (rms error)

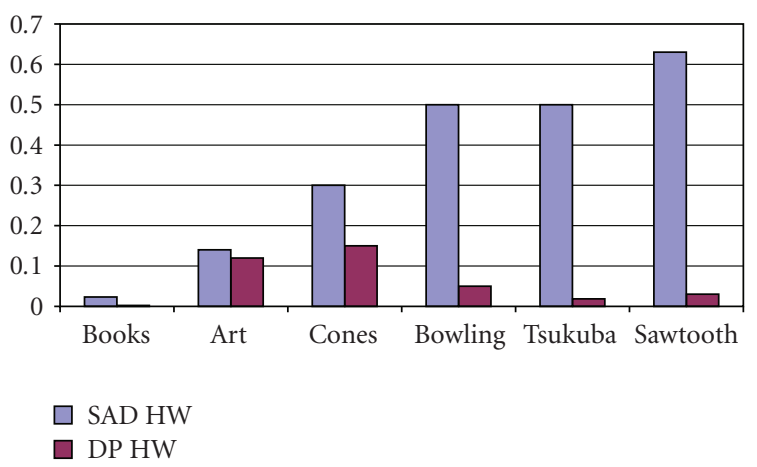

(b) Textureless regions (bad matches)

FIGURE 16: Errors computed in textureless regions for the various data-sets. (a) rms error, (b) percentage of bad matching pixels.

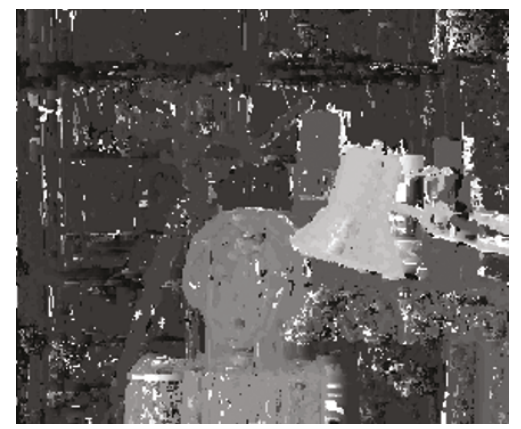

(a)

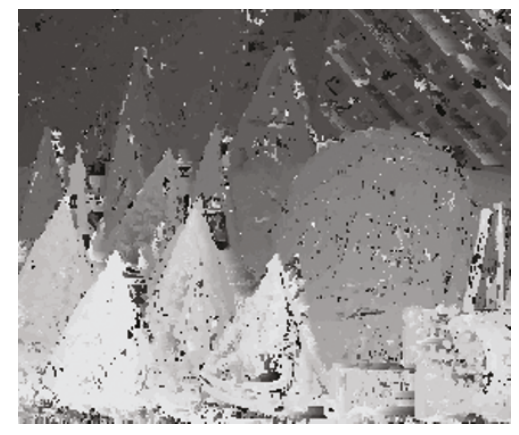

(c)

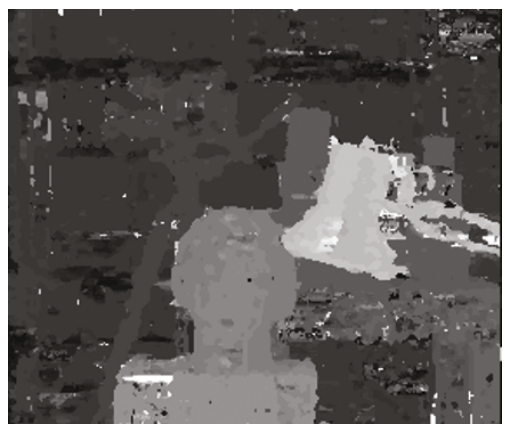

(b)

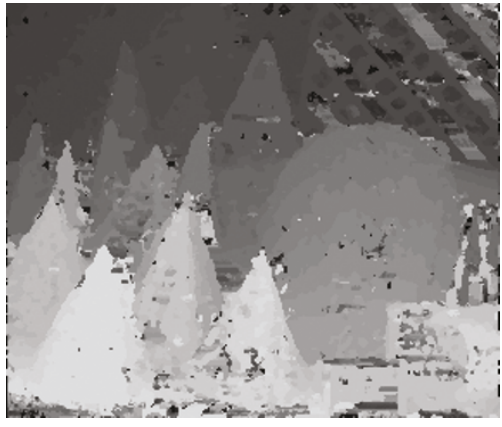

(d)

FIGURE 17: (b) and (d): Results from the SAD-based left-right consistency check. (a) and (c): Depth maps from the plain SAD system, shown for comparison.

380-pixels, the baseline is $0.125 \mathrm{~m}$, and the image centers are positioned on the pixel $(160,120)$ since the image resolution here is $320 \times 240$. No other calibration data are used in this experiment. The final projection in the real-world frame is given by a relative translation and rotation:

$$
\left(x_{i}, y_{i}, z_{i}\right)=\vec{R}+\operatorname{rot}_{\varphi}\left(X_{i}, Y_{i}, Z_{i}\right),
$$

where $\varphi$ is the angle between the reference frame of the stereo head and the world frame. $\vec{R}$ is the translation vector between the camera reference frame, and the world frame. In this experiment the stereo head is always horizontal.
The above procedure is applied indoors, in a typical office environment. Figure 22 is a panoramic picture of the captured scene. The stereo head rotates and traverses a total arc of about 60 degrees, recording the scene. The experiment lasts for ten seconds and at the end of this time a 3D cloud of points is projected in the world frame, reconstructing the scene according to (11)-(14).

A 3D graph of the resulting cloud of points is shown in Figure 23(a), as constructed using the DP hardware for the real-time computation of the depth map. The captured features correspond to the chairs in the middle and to the two bookshelves on the opposite and left wall, with respect to the 


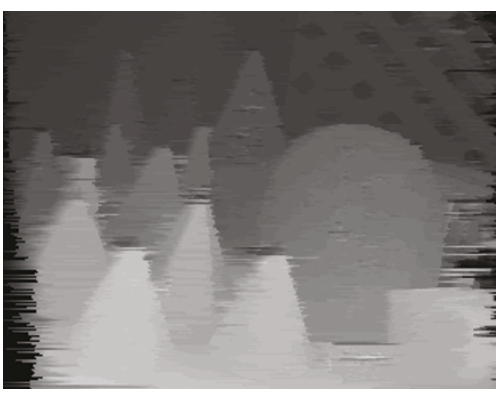

(a)

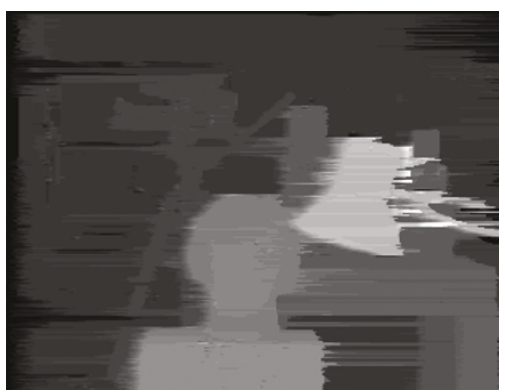

(c)

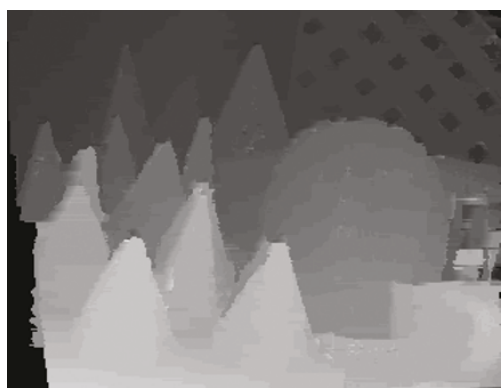

(b)

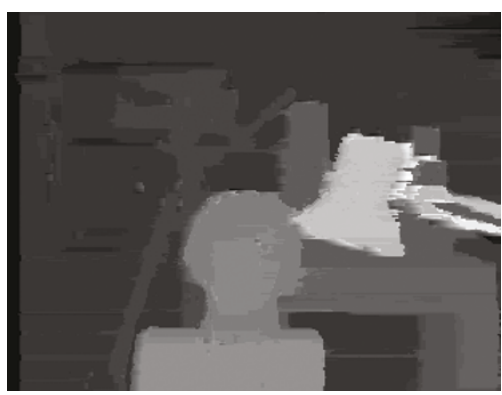

(d)

FIGURE 18: (b) and (d): Results from the DP system with interscanline support. (a) and (c): Results from the plain DP system shown for comparison.

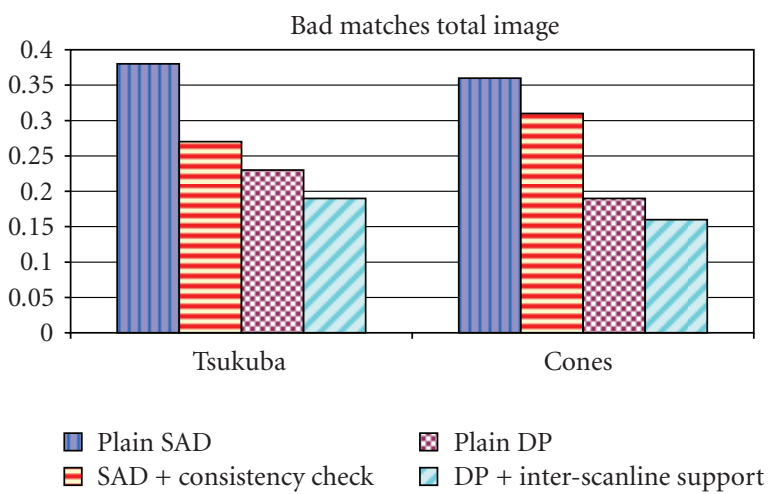

FIGURE 19: Comparison of the plain systems with the enhanced processors in terms of bad matching pixels.

camera. The desks shown in Figure 22 are not captured in the video sequence. A cross section presenting the $x$ - $z$ plane side view is shown in Figure 23(b), where points are stacked along the bookshelves on the right. The corresponding graphs constructed using the SAD hardware are shown in Figure 24.

Both DP and SAD techniques produce a reliable map of the environment, manifesting the main objects in their approximate positions. It is obvious, however, that the DP system results in more consistent correspondences and better localization of features. The SAD map includes a number of outlier points and presents fewer consistent features and a more dispersed cloud of points.

Factors that affect the result of the above experiment are (a) pixel noise due to camera limitations and poor illumination, (b) the lack of a full calibration of the stereo head, (c) inaccurate estimation of the camera angle $\varphi$ at each captured frame and (d) errors in the computation of the disparity map by the hardware systems. However, error sources a to $c$ are common in both DP and SAD experiments. Therefore, the differences in the maps presented in Figures 23 and 24 result solely from the differences in the hardware accelerators used to produce the disparity maps in real-time.

The mapping technique presented above can be incorporated as the measurement part in a fast simultaneous localization and mapping (Fast SLAM) stochastic procedure for autonomous robot navigation. In this way, the camera localization errors can be filtered out and an optimized solution for simultaneous robot pose and map estimation can be found in real-time.

\section{Comparison with Other Real-Time Stereo Accelerators}

Hardware acceleration of stereo processing is now established in many applications and a number of real-time stereo systems were presented in the literature in recent years. Several systems were built using FPGA devices, like the Xilinx Virtex series or the Altera Cyclone and Stratix families. As already mentioned in Section 1 most such systems are based on area correlation methods, using techniques like SAD $[6,7]$ and rank transforms [16]. Ambrosch and coauthors have recently implemented SAD-based stereo matching using FPGAs [17]. They also implemented SAD, rank and census transform with flexible block size and disparity range by reusing resources [18]. Their synthesis results show a high 


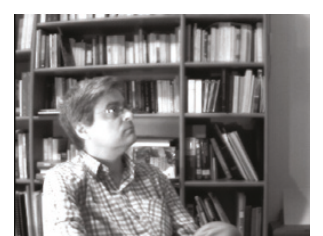

(a)

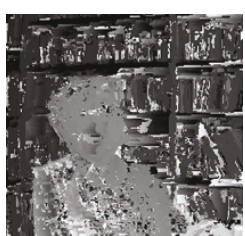

(b)

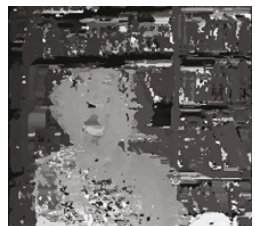

(c)

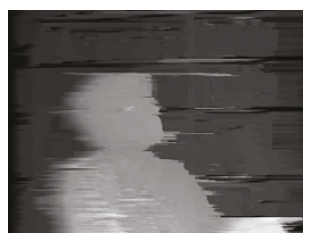

(d)

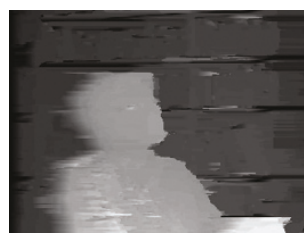

(e)

FIGURE 20: Processing of a real image with all types of processors: (a) right captured image, (b) processing with the plain SAD processor, (c) left-right consistency check with median filtering, and (d) plain DP processor (e) DP with interscanline support.

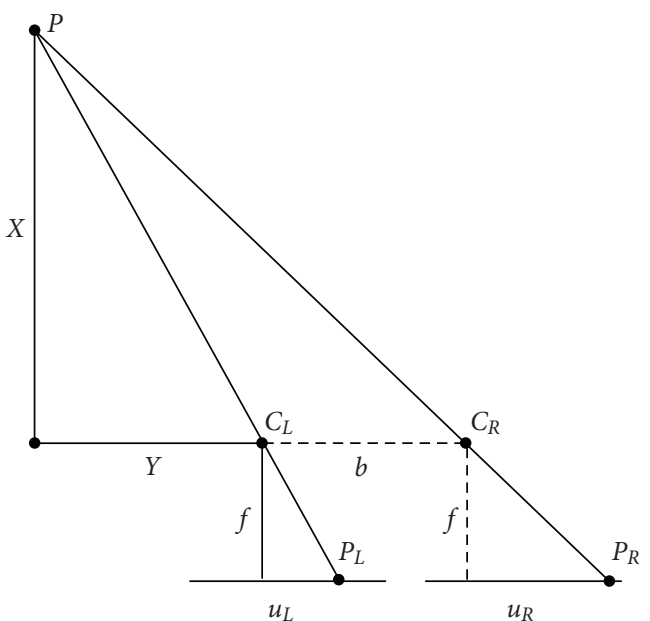

Figure 21: Top down view of two identical parallel cameras with focal length $f$ at distance $b$ to each other.

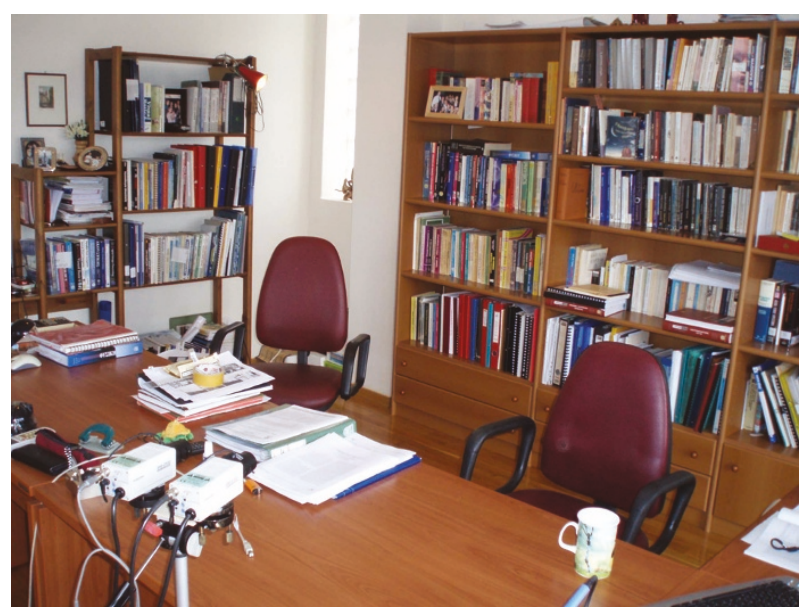

Figure 22: Panoramic view of the office premises where the mapping experiment was conducted.

resource usage of 106658 logic elements targeting a Stratix EP2S130 device, due to the high number of aggregations in a $9 \times 18$ block. Their quality assessment yields $61.12 \%$ and $79.85 \%$ correct matches for their SAD and census transform, respectively, while frame rates range to several hundreds.
Darabiha et al. [19] and Masrani and MacLean [20] implemented phase-based stereo in FPGAs using the equivalent of 70000 logic elements and about 800 Kbits of on-chip memory. Their system is built with Stratix S80 devices and supports a maximum disparity range of 128-pixels. A phasebased implementation is also reported in [21].

Global optimization VLSI architectures are just beginning to emerge. Recently, hardware-efficient algorithms for realizing belief propagation were proposed. Belief propagation (BP) is an effective technique for solving energy minimization problems in computer vision. It provides global optimality and good matching performance when applied to disparity estimation. However, it has great computational complexity and requires huge memory and bandwidth. Cheng et al. [22] propose a tile-based BP algorithm in order to overcome the memory and bandwidth bottlenecks. In a related article, Liang et al. [23] implemented the tile-based approach as well as a method for parallel message construction on a dedicated VLSI chip. The chip uses UMC $90 \mathrm{~nm}$ technology and consists of $2.5 \mathrm{M}$ gates (10 M transistors). It processes VGA images with 64-pixels maximum disparity at 27 frames per second.

The problem of mapping in hardware belief propagation for global stereo estimation is also addressed by Park and Jeong [24]. They implement a BP algorithm using a Xilinx XC2vp100 FPGA chip. They process 16 disparity levels and image resolution $256 \times 240$ at 25 frames per second, using 9564 slices (approximately 20000 LE) and more than 10 Mbits of memory partitioned in on-chip RAM blocks and external SRAM.

An FPGA implementation of a dynamic programming maximum likelihood algorithm has been presented by Sabihuddin and MacLean [25]. They use external memory for the two $N \times N$ stereo images and reduce the $N^{2}$ cost matrix elements to $2 \mathrm{~N}$ necessary elements. The partial cost matrix is stored in a $2 N \times 16$ bits RAM, while index values attributed to winning costs are stored in additional $N^{2} \times$ 2 bits on-chip memory. Exhaustive data processing occurs sequentially over all the pixels in the image scanline. Their design is much more complex and resource demanding than ours and can achieve $30 \mathrm{fps}$ applying several optimizations.

Software implementations of DP algorithms can achieve real-time matching at video rate by using coarse to fine approaches and exploiting the MMX assembly of contemporary CPUs [26]. In a similar recent trend, real-time performance of complex stereo algorithms has been boosted by the use of commodity graphics hardware [27]. Recent 


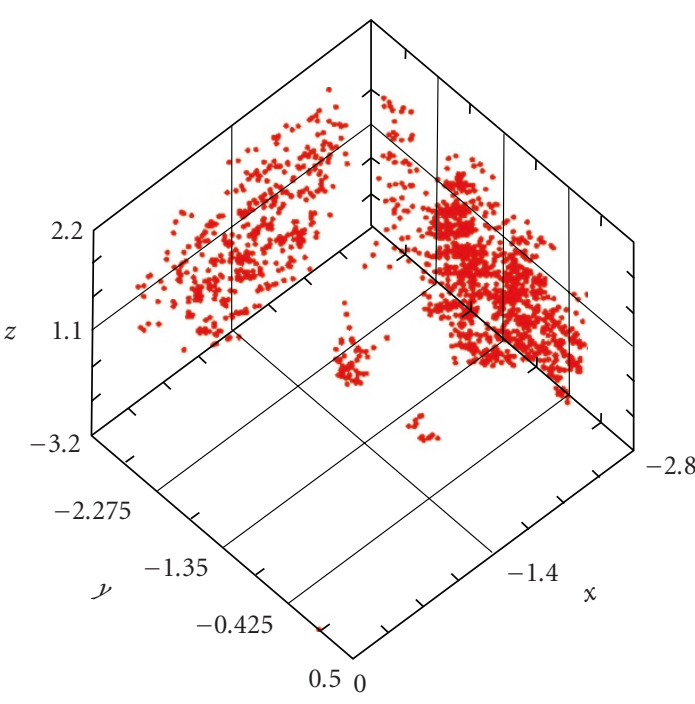

(a)

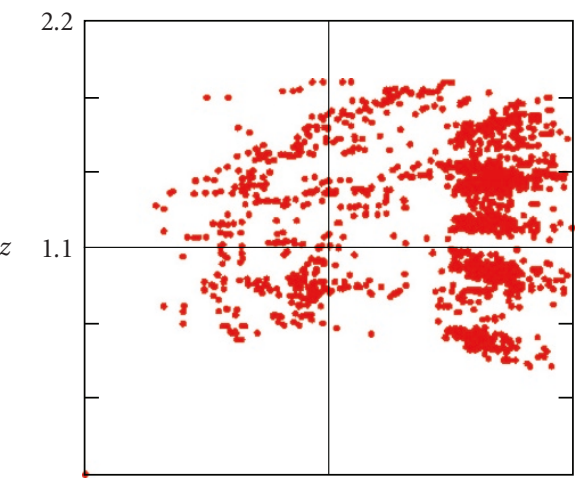

(b)

Figure 23: (a) Mapping result using the DP processor. (b) Side view ( $x$ - $z$ plane) of the office premises, with the book shelves visible on the right.

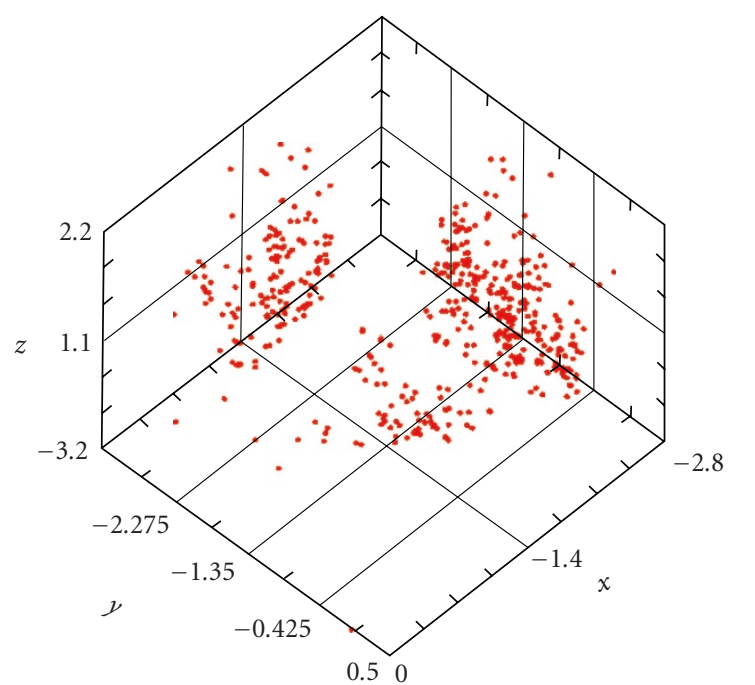

(a)

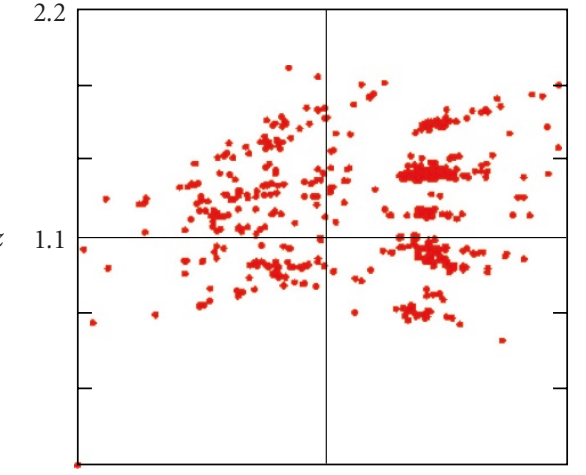

(b)

FIgURE 24: (a) Mapping using the SAD processor. (b) $x$ - $z$ plane of the map in (a).

advances exploit the parallel computational power within Graphics Processing Units (GPUs) which greatly exceeds that of CPUs. Local methods of stereo correspondence are more straightforward to implement using GPU [28, 29], however, several attempts have been made to map global techniques on the parallel programming model used by graphics units [30]. Methods based on belief propagation have produced the best results on GPUs [31], but they are computationally intensive and not very appropriate for realtime performance. Attempts to parallelize dynamic programming on graphics units have appeared in the literature and they offer a good balance between quality and speed $[32,33]$. In this respect, our proposed method to parallelize dynamic programming could be applied using parallel threads on a graphics unit, thus offering an alternative implementation. Using commodity graphics cards for stereo acceleration can be a flexible and cheap solution for PC-oriented applications, however, using dedicated hardware like our system is faster and more suitable for autonomous systems equipped with vision sensors.

In Tables 1 and 2 of the present article processing speeds and resource usage were presented for our own SAD and DP implementations on reconfigurable hardware. These data compare favorably with implementations reported in the aforementioned literature. The strong point of both SAD and DP architectures compared in this article is their 
full parallelism, resulting in one output disparity pixel at every clock count. Window-based parallelism is inherent in correlation methods like SAD and has been exploited by other systems too. In the case of the proposed DP system, parallelism is achieved by means of a novel state machine allowing cost-computation of $D$ states along the diagonal of the cost plane in one clock cycle. In this way, both systems evaluated in this article can perform in real-time and their normalized throughput is 50 Mpps. As shown in Table 1 when clocked at 100 and $50 \mathrm{MHz}$, respectively, they are able to process 162 and $81 \mathrm{fps}$, in full VGA resolution $(640 \times 480)$ and by these standards both systems compare favorably with other systems in the literature.

The overall quality of the depth maps produced by the DP hardware system is superior compared to SADbased implementations for comparable chip size. As shown in Section 5 the disparities computed by the DP hardware accelerator can be used successfully by a robotic system in order to reconstruct a map of its environment in real-time.

\section{Conclusion}

A comparative evaluation is presented of two different stereo processing systems implemented in reconfigurable hardware. A description of their hardware design is given. The two systems implement, respectively a method of local correlation using the measure of the Sum of Absolute Differences (SAD) and a maximum likelihood global technique based on Dynamic programming (DP) which optimizes the computation of disparities over the whole scan line. Enhancements of the two systems are also implemented in hardware, namely SAD-based left-right consistency check and interscanline support based on dynamic programming. Numerous test benches are used and depth maps are computed and compared to ground truth. The quality of the depth maps is measured in terms of rms error and percentage of bad matches, in regions rich with texture as well as in textureless regions of the image. The DP hardware processor is shown to produce fewer errors in almost all tests. Costcomputation using interscanline support minimizes errors present in the form of horizontal streaks.

Both processors are tested in a real-world 3D mapping experiment, using a video sequence in real-time. They are both able to map the scene, however, the DP processor produces the best results.

The present study shows that accelerating global techniques like dynamic programming with dedicated hardware can be a substantial improvement over traditional local correlation methods and can result in better real-time stereo vision systems in automotive, robotic, or other industrial applications.

\section{Acknowledgment}

J. Kalomiros wishes to acknowledge financial support provided by the Research Committee of the Technological Educational Institute of Serres, Greece, under Grant 71/13/710-2009.

\section{References}

[1] E. R. Davies, Machine Vision: Theory, Algorithms, Practicalities, Elsevier, San Diego, Calif, USA, 3rd edition, 2004.

[2] M. Z. Brown, D. Burschka, and G. D. Hager, "Advances in computational stereo," IEEE Transactions on Pattern Analysis and Machine Intelligence, vol. 25, no. 8, pp. 993-1008, 2003.

[3] S. Birchfield and C. Tomasi, "Depth discontinuities by pixelto-pixel stereo," in Proceedings of the IEEE International Conference on Computer Vision (ICCV '98), pp. 1073-1080, Mumbai, India, 1998.

[4] Y. Ohta and T. Kanade, "Stereo by intra- and inter-scanline search using dynamic programming," IEEE Transactions on Pattern Analysis and Machine Intelligence, vol. 7, no. 2, pp. 139154, 1985.

[5] I. J. Cox, S. L. Hingorani, S. B. Rao, and B. M. Maggs, "A maximum likelihood stereo algorithm," Computer Vision and Image Understanding, vol. 63, no. 3, pp. 542-567, 1996.

[6] Y. Miyajima and T. Maruyama, "A real-time stereo vision system with FPGA," in Field Programmable Logic and Applications, vol. 2778 of Lecture Notes in Computer Science, pp. 448457, Springer, Berlin, Germany, 2003.

[7] M. Hariyama, Y. Kobayashi, H. Sasaki, and M. Kameyama, "FPGA implementation of a stereo matching processor based on window-parallel-and-pixel-parallel architecture," IEICE Transactions on Fundamentals of Electronics, Communications and Computer Sciences (Japan), vol. E88-A, no. 12, pp. 35163521, 2005.

[8] O. Faugeras, B. Hotz, H. Matthieu, et al., "Real time correlation-based stereo: algorithm, implementations and applications," INRIA Technical Report 2013, INRIA, Lyon, France, 1993.

[9] J. A. Webb, "Implementation and performance of fast parallel multi-baseline stereo vision," in Proceedings of the DARPA Image Understanding Workshop, pp. 1005-1012, Washington, DC, USA, April 1993.

[10] J. A. Kalomiros and J. Lygouras, "Hardware implementation of a stereo co-processor in a medium-scale field programmable gate array," IET Computers and Digital Techniques, vol. 2, no. 5, pp. 336-346, 2008.

[11] A. L. Yuille and T. Poggio, "A generalized ordering constraint for stereo correspondence," Tech. Rep. A.I. Memo 777, AI Lab, MIT, Cambridge, Mass, USA, 1984.

[12] D. Scharstein and R. Szeliski, "High-accuracy stereo depth maps using structured light," in Proceedings of the IEEE Computer Society Conference on Computer Vision and Pattern Recognition (CVPR '03), vol. 1, pp. 195-202, Madison, Wis, USA, 2003.

[13] May 2009, http://cat.middlebury.edu/stereo/data.html.

[14] Y. Nakamura, T. Matsuura, K. Satoh, and Y. Ohta, "Occlusion detectable stereo-occlusion patterns in camera matrix," in Proceedings of the IEEE Computer Society Conference on Computer Vision and Pattern Recognition (CVPR '96), pp. 371378, 1996.

[15] D. Scharstein and R. Szeliski, "A taxonomy and evaluation of dense two-frame stereo correspondence algorithms," International Journal of Computer Vision, vol. 47, no. 1-3, pp. 7-42, 2002.

[16] J. Woodfill and B. Von Herzen, "Real-time stereo vision on the PARTS reconfigurable computer," in Proceedings of the 5th Annual IEEE Symposium on FPGAs for Custom Computing Machines, pp. 201-210, Napa Valley, Calif, USA, April 1997. 
[17] K. Ambrosch, M. Humenberger, W. Kubinger, and A. Steininger, "SAD-based stereo matching using FPGAs," in Embedded Computer Vision, Part II, B. Kisacanin, S. Bhattacharyya, and S. Chai, Eds., Springer, London, UK, 2009.

[18] K. Ambrosch, W. Kubinger, M. Humenberger, and A. Steininger, "Flexible hardware-based stereo matching," EURASIP Journal on Embedded Systems, vol. 2008, Article ID 386059, 12 pages, 2008.

[19] A. Darabiha, J. Rose, and W. J. MacLean, "Video-rate stereo depth measurement on programmable hardware," in Proceedings of the IEEE Computer Society Conference on Computer Vision and Pattern Recognition (CVPR '03), vol. 1, pp. 203-210, Madison, Wis, USA, June 2003.

[20] D. K. Masrani and W. J. MacLean, "A real-time large disparity range stereo-system using FPGAs," in Proceedings of the 4th IEEE International Conference on Computer Vision Systems (ICVS '06), pp. 13-20, January 2006.

[21] J. Díaz, E. Ros, S. Mota, E. M. Ortigosa, and B. del Pino, "High performance stereo computation architecture," in Proceedings of the International Conference on Field Programmable Logic and Applications (FPL '05), pp. 463-468, Tampere, Finland, August 2005.

[22] C.-C. Cheng, C.-K. Liang, Y.-C. Lai, H.-H. Chen, and L.-G. Chen, "Analysis of belief propagation for hardware realization," in Proceedings of the IEEE Workshop on Signal Processing Systems (SiPS '08), pp. 152-157, Washington, DC, USA, 2008.

[23] C.-K. Liang, C.-C. Cheng, Y.-C. Lai, L.-G. Chen, and H.-H. Chen, "Hardware efficient belief propagation," in Proceedings of the IEEE Computer Society Conference on Computer Vision and Pattern Recognition (CVPR '09), pp. 80-87, Miami, Fla, USA, June 2009.

[24] S. Park and H. Jeong, "High-speed parallel very large scale integration architecture for global stereo matching," Journal of Electronic Imaging, vol. 17, no. 1, Article ID 010501, 2008.

[25] S. Sabihuddin and W. J. MacLean, "Maximum likelihood stereo correspondence using field programmable gate arrays," in Proceedings of the 5th International Conference on Computer Vision Systems (ICVS '07), Bielefeld University, Bielefeld, Germany, March 2007.

[26] S. Forstmann, Y. Kanou, J. Ohya, S. Thuering, and A. Schmitt, "Real-time stereo by using dynamic programming," in Proceedings of the IEEE Computer Society Conference on Computer Vision and Pattern Recognition Workshops (CVPRW'04), vol. 3, p. 29, Washington, DC, USA, June 2004.

[27] S. Rogmans, "A generic framework for implementing realtime matching algorithms on the graphics processing unit," Barco Awards, pp. 1-10, 2007.

[28] M. Gong, R. Yang, L. Wang, and M. Gong, "A performance study on different cost aggregation approaches used in realtime stereo matching," International Journal of Computer Vision, vol. 75, no. 2, pp. 283-296, 2007.

[29] F. Tombari, S. Mattoccia, L. Di Stefano, and E. Addimanda, "Classification and evaluation of cost aggregation methods for stereo correspondence," in Proceedings of the 26th IEEE Conference on Computer Vision and Pattern Recognition (CVPR '08), pp. 1-8, June 2008.

[30] J. Gibson and O. Marques, "Stereo depth with a unified architecture GPU," in Proceedings of the IEEE Computer Society Conference on Computer Vision and Pattern Recognition Workshops (CVPRW'08), pp. 1-6, June 2008.
[31] A. Klaus, M. Sormann, and K. Karner, "Segment-based stereo matching using belief propagation and a self-adapting dissimilarity measure," in Proceedings of the 18th International Conference on Pattern Recognition (ICPR '06), vol. 3, pp. 15$18,2006$.

[32] J. Congote, J. Barandiaran, I. Barandiaran, and O. Ruiz, "Realtime dense stereo matching with dynamic programming in CUDA," in Proceedings of the 19th Spanish Congress of Graphical Informatics (CEIG '09), pp. 231-234, San Sebastian, Spain, September 2009.

[33] L. Wang, M. Liao, M. Gong, R. Yang, and D. Nister, "Highquality real-time stereo using adaptive cost aggregation and dynamic programming," in Proceedings of the 3rd International Symposium on 3D Data Processing, Visualization, and Transmission (3DPVT '06), pp. 798-805, Washington, DC, USA, 2006. 